\title{
DROPLET IMPACT IN ICING CONDITIONS - EXPERIMENTAL STUDY FOR WE 540
}

\begin{abstract}
The work presents investigation on the water droplet impingement at a substrate with three different surface coating. The experiments are carried out for two temperatures of the surface: $23^{\circ} \mathrm{C}$ (room temperature) and $-10^{\circ} \mathrm{C}$. The water droplet contact is recorded via ultra-fast camera and simultaneously via fast thermographic camera. The wetting properties are changing for subzero temperatures of substrates.
\end{abstract}

\section{Introduction}

The main objective of the presented work is to investigate an interaction of the water droplet with flat, impenetrable surface in icing conditions. Such conditions can occur in everyday life (e.g., vehicle icing), during the use of industrial installations (e.g. the high voltage lines), on the surfaces of vessels and especially on the surfaces of airplanes, in the case when life threatening situations may occur because of icing. In recent years, researchers in material sciences and hydrodynamics showed interesting possibilities to eliminate icing [1-4], without the use of the so-called traditional de-icing methods (e.g., heating of surfaces). The investigations presented in this work try to identify basic phenomena occurring during icing and to recognize basic issues that take place during impingement $[5,6]$. The dynamic experiments, described in this work, in particular the spreading and the relaxation phases (see Fig. 1) follow the impingement scenarios firstly proposed by Rioboo [7]. During last years, the kinematic and the spreading phases have been widely described for the droplet impingement for room temperatures. The

\footnotetext{
${ }^{1}$ Warsaw University of Technology, Institute of Aeronautics and Applied Mechanics, Warsaw, Poland.Email:mremer@meil.pw.edu.pl

${ }^{2}$ Faculty of Chemistry, Department of Materials Technology and Chemistry, University of Lodz, Pomorska 163, 90-236 Lodz, Poland
} 
maximum diameter during spreading given by Laan et.al [8] and dependence of the droplet radius in the function of time described in the work by Stapelbroek [9] are not dependent on the surface properties.

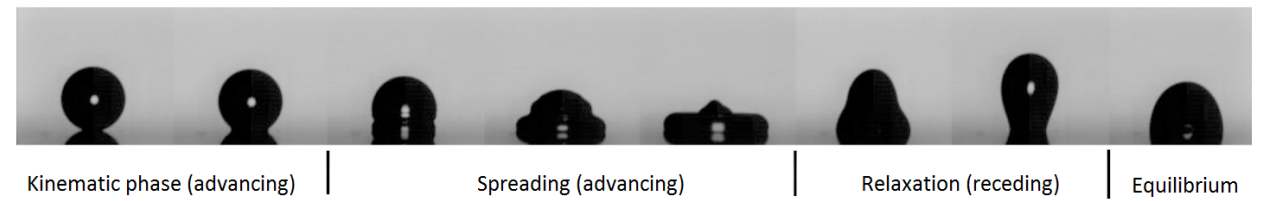

Fig. 1. Phases of the droplet deformation proposed $(\mathrm{We}=10)$

\section{Methodology}

The dynamic droplet contact with surfaces was studied at a specially designed experimental stands of the Department of Aerodynamics. Two different types of experiments were carried out - at the room temperatures and at subzero temperatures. In the first one, the droplet, the air and the sample (the plate) had the same temperature, usually about $20^{\circ} \mathrm{C}$. The results obtained from the first series were used as a benchmark for the subzero measurements. The second "climatic" configuration, allowed one for the measurements during which the temperature of the droplet, the air, the sample as well the humidity level of the surrounding air were controlled, both for the room and the subzero temperatures. For both installations, similar velocities of the droplet were obtained to decrease the number of parameters resulting from the droplet impingement.

The snapshots of the droplet during the impingement process were obtained by the use of a super-fast camera (Photron Fastcam SA5) mounted at different angles to obtain full information about the phenomenon. The additional side view was obtained from the thermographic camera Flir model X6580sc.

Apart from the two high-speed cameras (one thermographic), the test-stand included a Peltier cell connected to the CPU heat exchanger (Fig. 2). Such configuration can maintain the temperature of the surface within the range from $-10^{\circ} \mathrm{C}$ to $25^{\circ} \mathrm{C}$. The sample was placed in a closed chamber filled with nitrogen to exclude humidity, with a closed circuit used to cool down the whole test section. To generate droplets, a syringe equipped within laser-optical gate was used. The laser optical gate generated the TTL normalised signal, while the droplet passed the laser beam, enabling the simultaneous recording of the impingement phenomena (with the use of both cameras).

In the first series of experiments, the temperature of the air, water and the surface was kept at $23^{\circ} \mathrm{C}$, while the relative humidity was $22 \%$. In the second series, the sample was cooled to $-10^{\circ} \mathrm{C}$, while other parameters remained the same as in the first series (see Table 1). 


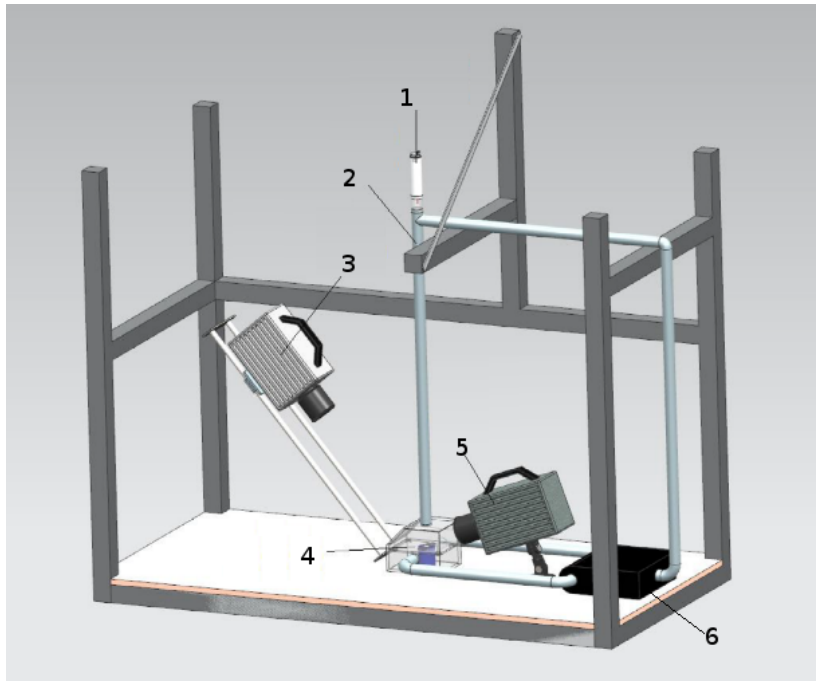

Fig. 2. Experimental stand for the test with heat exchange $(1-$ syringe with optical trigger, 2 - cooling circuit, 3 - fast camera, 4 - test chamber with Peltier cell, 5 - thermographic camera, 6 - heat exchanger)

Table 1.

Climate parameters obtained for each series of experiments

\begin{tabular}{|l|c|r|}
\hline Experiment series & I & \multicolumn{1}{c|}{ II } \\
\hline Chamber temperature & $23^{\circ} \mathrm{C}$ & $23^{\circ} \mathrm{C}$ \\
\hline Surface temperature & $23^{\circ} \mathrm{C}$ & $-10^{\circ} \mathrm{C}$ \\
\hline Droplet temperature & $23^{\circ} \mathrm{C}$ & $23^{\circ} \mathrm{C}$ \\
\hline Relative humidity & $22 \%$ & $22 \%$ \\
\hline
\end{tabular}

\section{Surfaces used in experiments}

In the present work, experiments for three different smooth surfaces were carried out. The surfaces had wetting properties ranging from slightly hydrophilic to highly hydrophobic. The surfaces were manufactured at Lodz University of Technology (see [10]), with only chemical functionalization. The surfaces were free of any micro-structures to avoid water condensation in the pores. While manufacturing the substrates, firstly the cover glass slides $(22 \times 22 \mathrm{~mm}, 0.15 \mathrm{~mm}$ thick, Knittel Glass, Braunschweig, Germany) were purged at first with dry air. Next, consecutive layers were deposited on the glass or silicon substrates: binding layer of chromium, hydrophilic layer of gold and hydrophobic layers of either decanethiol (DT) or fluorinated decanethiol (FDT) (Fig. 3) [10].

Decanethiol (97\%) (DT) and 1H,1H,2H,2H-perfluorodecanethiol (97\%) (FDT) were obtained from Sigma-Aldrich and were used without further purification. The coatings used in this work were supposed to reduce wettability of surfaces. The 


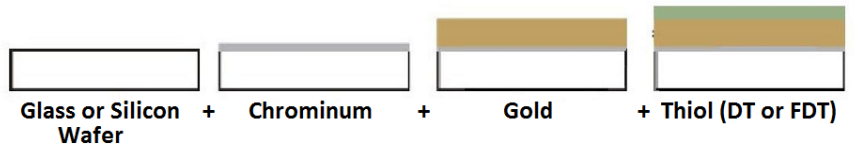

Fig. 3. Scheme of substrate coverage [10]

pure gold surface (without any chemical surfactants described above) was used as a reference one. Table 2 presents contact angles and its hysteresis for the selected surfaces. Deposition of DT or FDT coatings onto the Au layers resulted in RMS values of 1.5-1.6 $\mathrm{nm}$ for $\mathrm{Si}$ substrates and $4.4-6.2 \mathrm{~nm}$ for glass substrates. Overall, the surfaces of the coatings were microscopically smooth, with small variations of roughness at a level of a few nanometres. Such a mechanical property of the surface has minimised existence of the water vapour inside the pores of the surface. In this work, the glass substrates were used for all experiments.

Table 2.

Contact angles for specified surfaces (glass base), measured by sessile drop method [10]

\begin{tabular}{|l|r|r|r|}
\hline Surface & $\Theta_{A}$ & $\Theta_{R}$ & $\Delta \Theta$ \\
\hline $\mathrm{Au}$ & 85 & 46 & 39 \\
\hline $\mathrm{Au}+\mathrm{DT}$ & 108 & 104 & 4 \\
\hline $\mathrm{Au}+\mathrm{FDT}$ & 119 & 101 & 18 \\
\hline
\end{tabular}

\section{Results of measurements at room temperature}

For all three tested surfaces, at least six impingement videos were recorded to confirm repeatability of the results, since the homogeneity of the surface was confirmed in previous work [10]. Table 3 shows the parameters describing droplet, which are typical for such experiments. For all recorded videos, the droplet initial diameter varied between $1.95 \mathrm{~mm}$ and $2.25 \mathrm{~mm}$. Due to the limitation of the frame rate, the very first phase of movement - the kinematic one - could not be observed.

Parameters of droplets during impingement

Table 3.

\begin{tabular}{|l|c|c|c|c|c|}
\hline Surface & $D[\mathrm{~mm}]$ & $d_{\max }^{*}$ & $u[\mathrm{~m} / \mathrm{s}]$ & $\mathrm{We}$ & $\mathrm{Re}$ \\
\hline $\mathrm{Au}$ & 2,2 & 4,50 & 3,96 & 476 & 8654 \\
\hline $\mathrm{Au}+\mathrm{DT}$ & 2,15 & 4,51 & 4,24 & 534 & 9066 \\
\hline $\mathrm{Au}+\mathrm{FDT}$ & 2,1 & 4,57 & 4,01 & 467 & 8378 \\
\hline
\end{tabular}

This was due to the relatively high speed of impingement. According to the work by Rioboo [7], additional parameters were calculated to preserve proper description of the droplet impingement phases.

The non-dimensional time

$$
t^{*}=\frac{t u}{D_{0}}
$$


and spread factor

$$
d^{*}=\frac{d}{D_{0}}
$$

are defined by the above formulae, where $u$ - is the impact velocity, $d$ - is the instant droplet diameter and $D_{0}-$ is the initial droplet diameter. For the first frame, after droplet contact, the dimensionless time was around 0.19 , while the movement phase lasted for a shorter time (around $t^{*}=0.1$ ).

To ensure that the droplet would not cool down during the free fall, the velocity response time (3)

$$
\tau_{v}=\frac{\rho_{d} d^{2}}{18 \mu}
$$

and the thermal response time (4) given by Crowe [11] were calculated

$$
\tau_{T}=\frac{\rho_{d} c_{d} d^{2}}{12 k_{c}},
$$

where $\rho_{d}$ is the droplet density, $d$ is the droplet diameter, $\mu$ is the droplet dynamic viscosity, $c_{d}$ is the droplet specific heat and $k_{c}$ is the continuum (air) thermal conductivity.

For the droplet diameter equal $\sim 2.2 \mathrm{~mm}$, the water and air temperature $\sim 23^{\circ} \mathrm{C}$, the calculated values of $\tau_{v}$ and $\tau_{T}$ were equal, respectively, $\sim 14$ seconds and $\sim 60$ seconds, and were much greater than the time of the experiment.

The following advancing phase of the droplet impingement is shown in Fig. 4. For all surfaces, the time to reach maximum diameter is the same and is equal to 1.7 ms. The maximum diameter values are shown in Table 3. It can be observed that, for all surfaces, the maximum diameter does not vary much, similarly as reported by other authors [7]. Nevertheless, the observed small differences should be studied in detail a the wider range of surfaces characterised by different contact angles.

However, distinct differences can be observed (see Fig. 5) in the relaxation phase (receding phase). It can be clearly seen that, for the tested surfaces, the velocity of retraction is different. In work by Remer et.al [10] the distinction in behaviour was also noticeable, for different range of We number (We $=5 \div 10$ ). For the reference case (Au and Au+DT surfaces) the time to reach final diameter is specified in Fig. 5. However, for more hydrophobic (Au+FDT) substrate, partial bouncing occurs and that is why the specified time is reported for the moment when the water column appears.

Schematic description of partial bouncing is shown in Fig. 6, obtained by thermographic camera for the Au+FDT surface. It appeares that the camera frame rate is enough to observe consecutive phases of the droplet impingement and the field of temperature on the water droplet surface.

The important observed fact is the decrease of relaxation time, while the contact angle is getting larger. This means that it takes longer time to retract for the hydrophilic and shorter for the hydrophobic surfaces. It should be also mentioned that, for more hydrophobic surfaces, the receding phase is much more dynamic. 

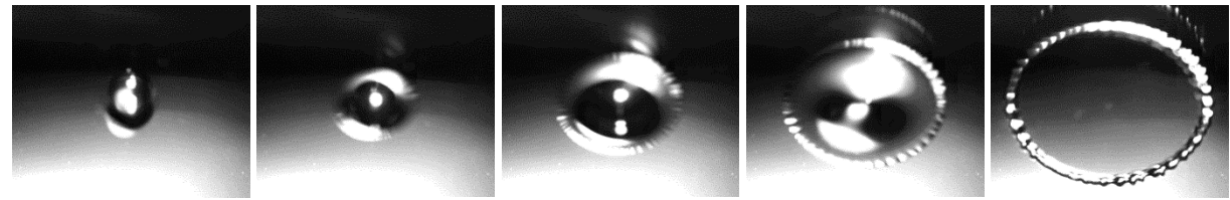

$t=0.1 \mathrm{~ms}$

$t^{*}=0.18$

$t=0.2 \mathrm{~ms}$

$t=0.4 \mathrm{~ms}$

$t=0.7 \mathrm{~ms}$

$t=1.7 \mathrm{~ms}$
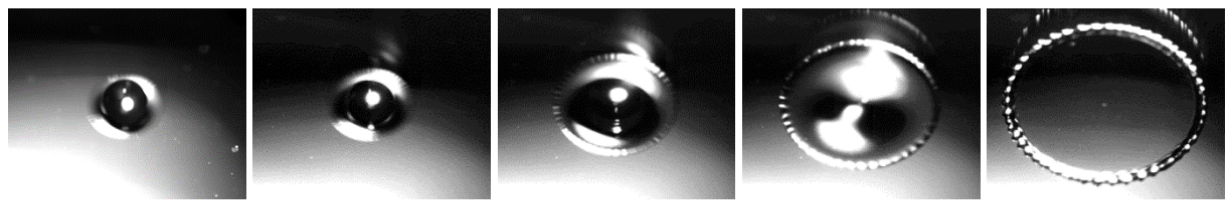

$t=0.1 \mathrm{~ms}$

$t^{*}=0.2$

$t=0.2 \mathrm{~ms}$

$t=0.4 \mathrm{~ms}$

$t=0.7 \mathrm{~ms}$

$t=1.7 \mathrm{~ms}$
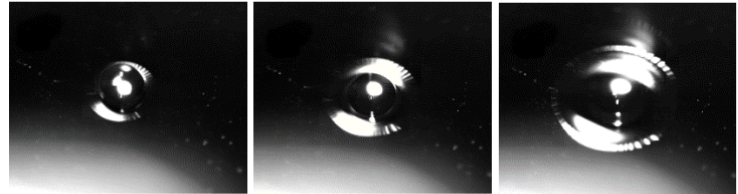

$t=0.1 \mathrm{~ms}$

$t=0.2 \mathrm{~ms}$

$t=0.4 \mathrm{~ms}$

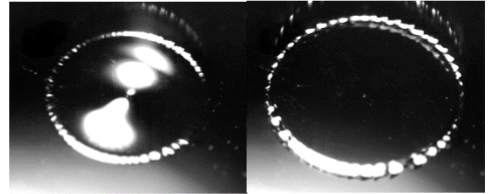

$t=0.7 \mathrm{~ms}$

$t=1.7 \mathrm{~ms}$

Fig. 4. Sequence of images for the droplet impingement for surfaces: Au (upper), Au+DT (middle),

$\mathrm{Au}+\mathrm{FDT}$ (bottom) in function of time for advancing phase. Temperature of surface $T=23^{\circ} \mathrm{C}$

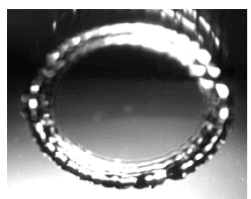

$t=2.7 \mathrm{~ms}$

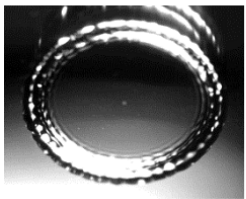

$t=2.7 \mathrm{~ms}$

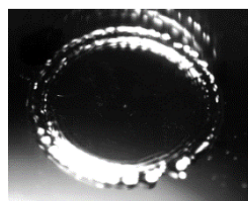

$t=2.7 \mathrm{~ms}$
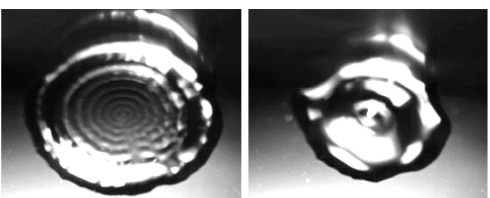

$t=10.4 \mathrm{~ms}$

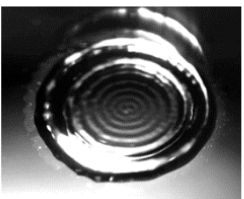

$t=5 \mathrm{~ms}$

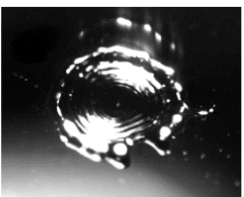

$t=5 \mathrm{~ms}$

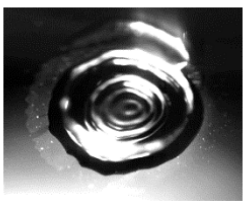

$t=7 \mathrm{~ms}$

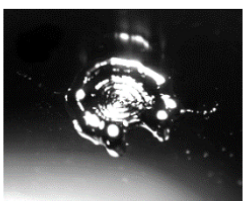

$t=6 \mathrm{~ms}$
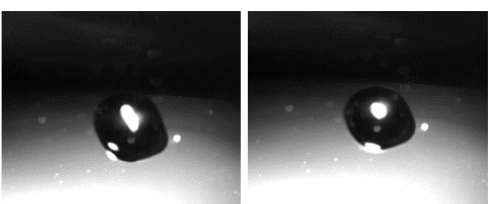

$t=25.3 \mathrm{~ms}$

$t=33.8 \mathrm{~ms}$

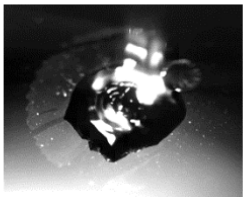

$t=10.4 \mathrm{~ms}$

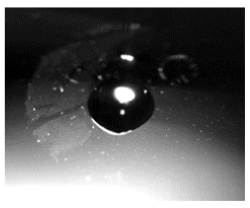

$t=25.3 \mathrm{~ms}$

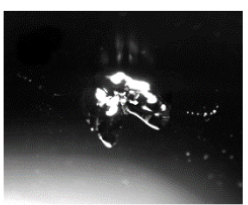

$t=7 \mathrm{~ms}$

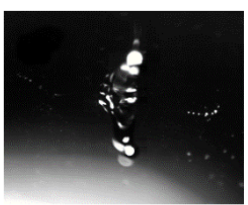

$t=10.4 \mathrm{~ms}$

Fig. 5. Sequence of images for the droplet impingement for surfaces: Au (upper), Au+DT (middle), $\mathrm{Au}+\mathrm{FDT}$ (bottom) in function of time for the receding phase. Temperature of surface $T=23^{\circ} \mathrm{C}$ 


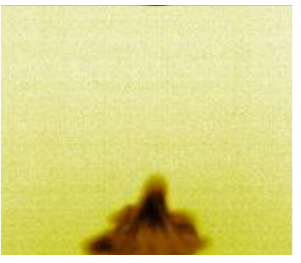

$t=6.9 \mathrm{~ms}$

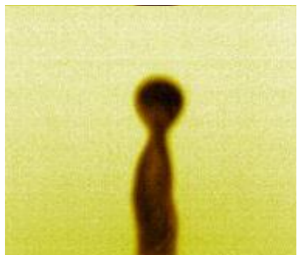

$t=12 \mathrm{~ms}$

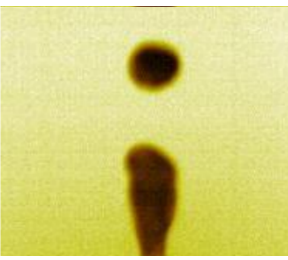

$t=15.4 \mathrm{~ms}$

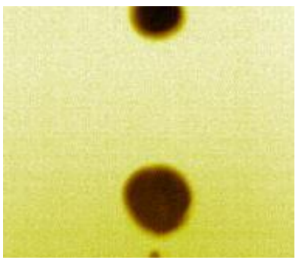

$t=20.6 \mathrm{~ms}$

Fig. 6. Pictorial sequence of images for the droplet partial bouncing for surface Au+FDT in function of time. Obtained by thermographic camera

\section{Measurements results at $-10^{\circ} \mathrm{C}$ temperature}

The icing measurements were carried out in the conditions described in Section 2 and the results for velocities and diameters are shown in Table 4.

Table 4.

Parameters of droplets during impingement in icing experiment

\begin{tabular}{|l|c|c|c|c|c|}
\hline Surface & $D[\mathrm{~mm}]$ & $d_{\text {max }}^{*}$ & $u[\mathrm{~m} / \mathrm{s}]$ & $\mathrm{We}$ & $\mathrm{Re}$ \\
\hline $\mathrm{Au}$ & 2,15 & 4,65 & 4,29 & 545 & 9161 \\
\hline $\mathrm{Au}+\mathrm{DT}$ & 2,1 & 4,67 & 4,35 & 548 & 9080 \\
\hline $\mathrm{Au}+\mathrm{FDT}$ & 2,0 & 4,85 & 4,31 & 513 & 8570 \\
\hline
\end{tabular}

In analogy to the room temperature experiments, impingement was repeated for the surfaces cooled down to $-10^{\circ} \mathrm{C}$. For all three substrates, the time to reach maximum diameter was the same with only small differences (Fig. 7). However, when results were compared to room temperature configuration, small instabilities on the outer rim of the droplet were identified for the maximum diameter (see Fig. $4 t=1.8 \mathrm{~ms}$ ). For the surface $\mathrm{Au}$ and Au+DT there were about 30 fingers while for the surface Au+FDT there were about 26. There was also a difference in the scale of fingering (the fingers did not occur for room temperature experiment). Such instabilities were described earlier by Stanley et.al [12] and took place when droplet exceeded certain level of Weber number.

Additionally, only for the hydrophobic surface (Au+FDT) the phenomenon called prompt splash appeared, during which small amounts of water were ejected radially from the surface, with very high speed (Fig. 8) [13].

While considering the receding phase, partial freezing can be observed. As a result, for all substrates the retraction to the centre of impingement (final diameter) was slower than for the ambient temperature - almost 10 times slower for Au. For the Au+FDT surface it was only 2 times longer (see Fig. 9). While the droplet retracts, it removes the moisture condensed at the surface.

Simultaneously the thermographic camera recorded pictures which allowed qualitative analysis of the temperature distribution (the darker, the colder). The thermographic camera was focused on the surface and the emissivity of the camera 


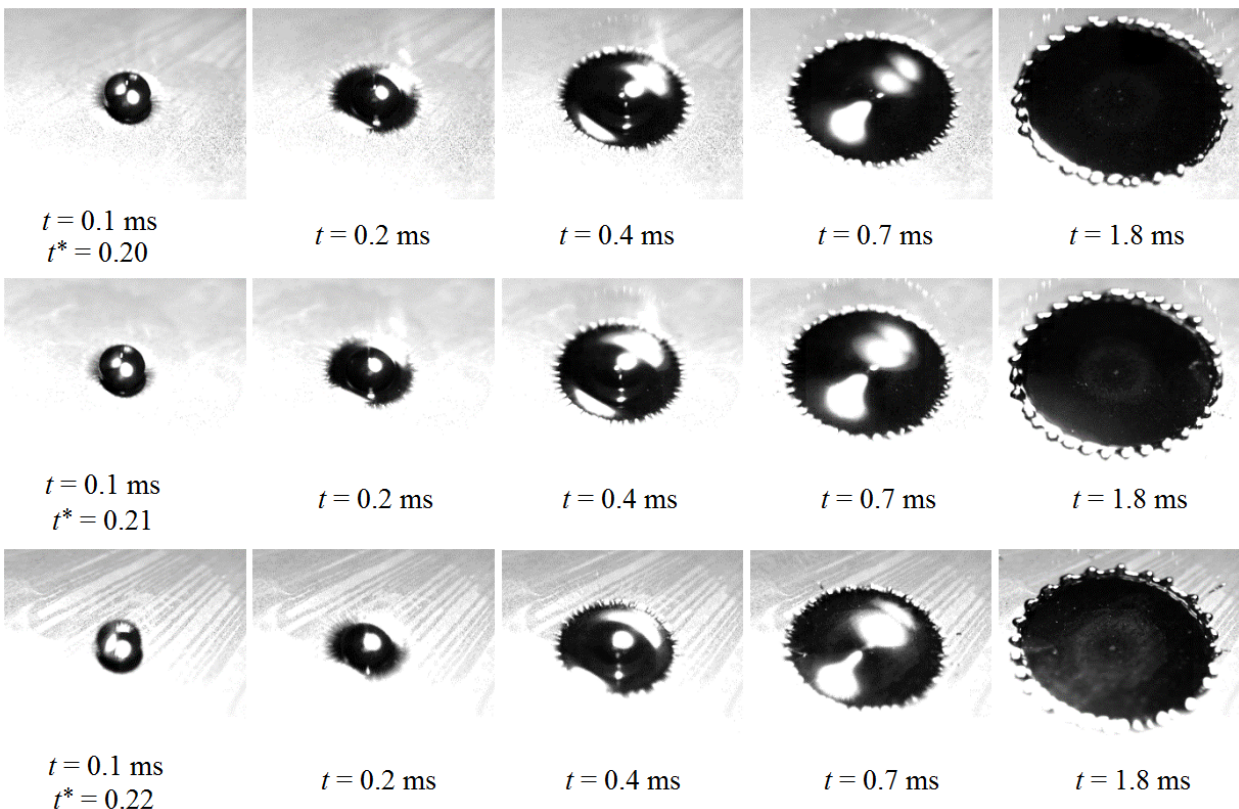

Fig. 7. Sequence of images for the droplet impingement for surfaces : Au (upper), Au+DT (middle), $\mathrm{Au}+\mathrm{FDT}$ (bottom) in function of time for advancing phase. Temperature of the surface $T=-10^{\circ} \mathrm{C}$

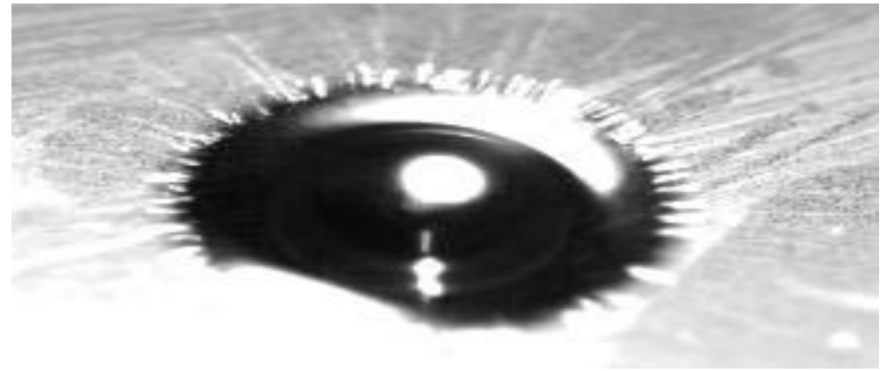

Fig. 8. Picture of the prompt splash for the Au+FDT surface at $T=-10^{\circ} \mathrm{C}$

was set to validate the surface temperature. However, the camera frame rate and its thermal resolution gave possibility to record relative temperatures distribution of the droplet. Thanks to the temperature distribution, convection movement (mixing) within the droplet can be observed (Au+FDT Fig. 10). Such phenomena can be only seen on the Au+FDT surface. The droplet warmed up the surface and removed the cold moisture, while base of the droplet stayed colder (see black colour at the droplet base - Fig. 10, $t=27.8 \mathrm{~ms}$ ). The obtained results may be useful for determining the timescales both for mechanical and thermodynamically regimes $[11,14]$. 


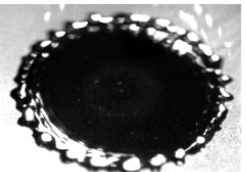

$t=2.8 \mathrm{~ms}$

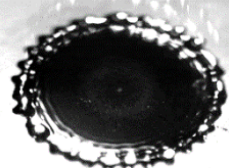

$t=2.8 \mathrm{~ms}$

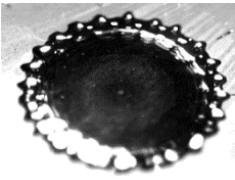

$t=2.8 \mathrm{~ms}$

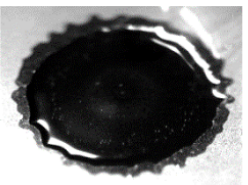

$t=19.4 \mathrm{~ms}$

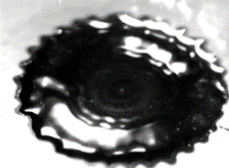

$t=6 \mathrm{~ms}$

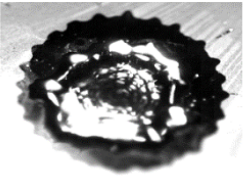

$t=6 \mathrm{~ms}$

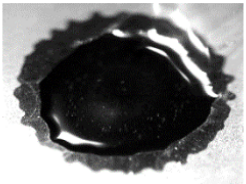

$t=35 \mathrm{~ms}$

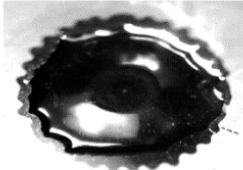

$t=10 \mathrm{~ms}$

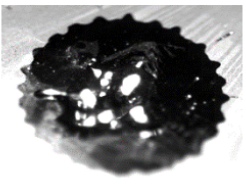

$t=9 \mathrm{~ms}$

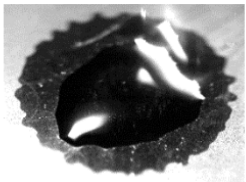

$t=57.4 \mathrm{~ms}$

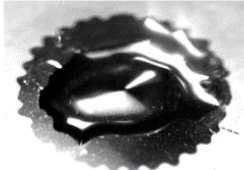

$t=19.4 \mathrm{~ms}$

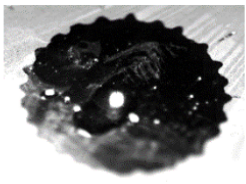

$t=13 \mathrm{~ms}$

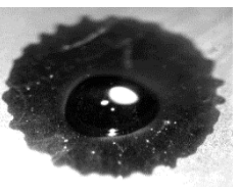

$t=257.1 \mathrm{~ms}$

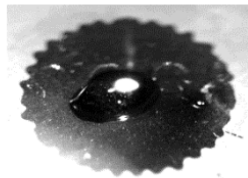

$t=57.4 \mathrm{~ms}$

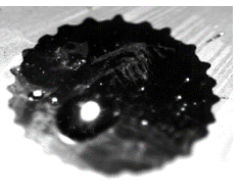

$t=19.4 \mathrm{~ms}$

Fig. 9. Sequence of images for the droplet impingement, for surfaces: Au (upper), Au+DT (middle), $\mathrm{Au}+\mathrm{FDT}$ (bottom) in function of time for the receding phase. Temperature of the surface $T=-10^{\circ} \mathrm{C}$

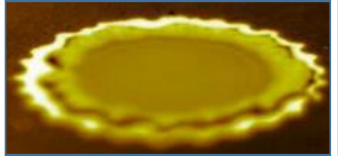

$t=3.8 \mathrm{~ms}$

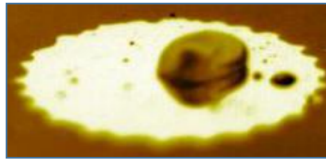

$t=14.1 \mathrm{~ms}$

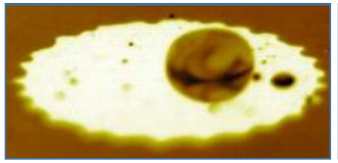

$t=24.4 \mathrm{~ms}$

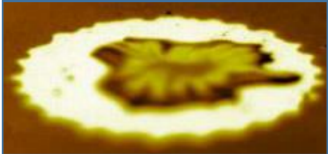

$t=7.2 \mathrm{~ms}$

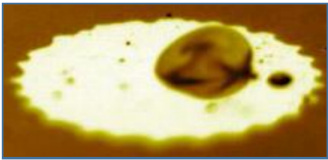

$t=17.5 \mathrm{~ms}$

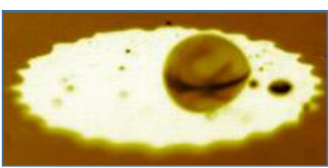

$t=27.8 \mathrm{~ms}$

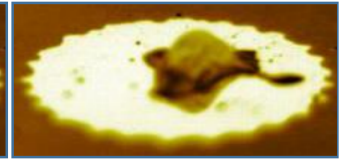

$t=10.7 \mathrm{~ms}$

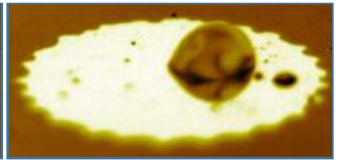

$t=21.0 \mathrm{~ms}$

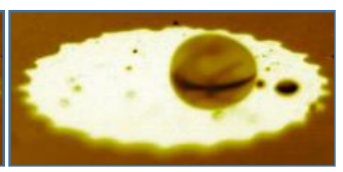

$t=31.2 \mathrm{~ms}$

Fig. 10. Relative temperature distribution on the droplet surface Au+FDT (the darker, the colder)

\section{Conclusions}

The dynamic interaction of water droplet with smooth surfaces was investigated experimentally. Three surfaces with different chemical coatings were tested at room temperature $23^{\circ} \mathrm{C}$ and for $-10^{\circ} \mathrm{C}$. The first two phases: the kinematic and the spreading (advancing) one are similar for both room and subzero temperatures. However, for the temperature of substrate below $0^{\circ} \mathrm{C}$, finger instabilities appear for 
all substrates for maximum diameter. These instabilities vary in size and the number of fingers depending on the surface tested. Additionally, for the most hydrophobic surface, prompt splash appears, in which small amount of water is ejected radially with a very high speed.

For the relaxation phase, only partial icing was observed for all tested surfaces. The time to reach the final diameter (with no movement) has increased for $\mathrm{Au}$ surface almost 10 times (up to $260 \mathrm{~ms}$ ), while for the surface Au+FDT (most hydrophobic), it has doubled (up to $\sim 20 \mathrm{~ms}$ ). The pictures obtained from the thermographic camera allowed us to obtain surface temperature distributions. For the most hydrophobic surface and the temperature of $-10^{\circ} \mathrm{C}$, the convection movement inside the droplet was observed.

The presented measurements were carried out on a newly reconfigured test stand of the Department of Aerodynamics. It must be concluded that more accurate temperature measurements are necessary to fully identify the occurring freezing phenomena. Similarly, more diversified substrates should be further investigated.

\section{Acknowledgements}

This work was supported by the National Science Centre of Poland under grant no. UMO2012/05/B/ST8/02876.

Manuscript received by Editorial Board, September 07, 2016; final version, March 18, 2017.

\section{References}

[1] A. Alizadeh, V. Bahadur, S. Zhong, W. Shang, R. Li, J. Ruud, M. Yamada, L. Ge, A. Dhinojwala, and M. Sohal. Temperature dependent droplet impact dynamics on flat and textured surfaces. Applied Physics Letters, 100(11):111601, 2012. doi: 10.1063/1.3692598.

[2] M. Nosonovsky and V. Hejazi. Why superhydrophobic surfaces are not always icephobic. ACS Nano, 6(10):8488-8491, 2012. doi: 10.1021/nn302138r.

[3] K.K. Varanasi, T. Deng, M. Hsu, and N. Bhate. Hierarchical superhydrophobic surfaces resist water droplet impact. In Technical Proceedings of the 2009 NSTI Nanotechnology Conference and Expo, Houston, Texas, USA, 3-7 May 2009. Nano Science and Technology Institute. http://hdl.handle.net/1721.1/64767.

[4] L. Mishchenko, B. Hatton, V. Bahadur, J.A. Taylor, T. Krupenkin, and J. Aizenberg. Design of ice-free nanostructured surfaces based on repulsion of impacting water droplets. ACS Nano, 4(12):7699-7707, 2010. doi: 10.1021/nn102557p.

[5] R. Ramachandran, K. Sobolev, and M. Nosonovsky. Dynamics of droplet impact on hydrophobic/icephobic concrete with the potential for superhydrophobicity. Langmuir, 31(4):1437-1444, 2015. doi: 10.1021/la504626f.

[6] T. Bobinski, G. Sobieraj, K. Gumowski, J. Rokicki, M. Psarski, J. Marczak, and G. Celichowski. Droplet impact in icing conditions - the influence of ambient air humidity. Archives of Mechanics, 66(2):127-142, 2014. http://am.ippt.pan.pl/index.php/am/article/view/v66p127.

[7] R. Rioboo, M. Marengo, and C. Tropea. Time evolution of liquid drop impact onto solid, dry surfaces. Experiments in Fluids, 33(1):112-124, 2002. doi: 10.1007/s00348-002-0431-x. 
[8] N. Laan, K.G. de Bruin, D. Bartolo, C. Josserand, and D. Bonn. Maximum diameter of impacting liquid droplets. Physical Review Applied, 2(4):044018, 2014. doi: 10.1103/PhysRevApplied.2.044018.

[9] B.B.J. Stapelbroek, H.P. Jansen, E.S. Kooij, J.H. Snoeijer, and A. Eddi. Universal spreading of water drops on complex surfaces. Soft Matter, 10(15):2641-2648, 2014. doi: $10.1039 / \mathrm{c} 3 \mathrm{sm} 52464 \mathrm{~g}$.

[10] M. Remer, M. Psarski, K. Gumowski, J. Rokicki, G. Sobieraj, M. Kaliush, D. Pawlak, and G. Celichowski. Dynamic water contact angle during initial phases of droplet impingement. Colloids and Surfaces A: Physicochemical and Engineering Aspects, 508:57-69, 2016. doi: 10.1016/j.colsurfa.2016.08.028.

[11] C.T. Crowe. Multiphase Flow Handbook, volume 59 of Mechanical and Aerospace Engineering Series. CRC Press, 2005.

[12] C. Stanley, R. Jackson, N. Karwa, and G. Rosengarten. The effects of surface wettability on droplet fingering. In The Proceedings of the 19th Australasian Fluid Mechanics Conference, Melbourne, Australia, 8-11 December 2014. Paper No. 49.

[13] A. Latka, A. Strandburg-Peshkin, M.M. Driscoll, C.S. Stevens, and S.R. Nagel. Creation of prompt and thin-sheet splashing by varying surface roughness or increasing air pressure. Physical Review Letters, 109(5):054501, 2012. doi: 0.1103/PhysRevLett.109.054501.

[14] T.G. Myers, J.P.F. Charpin, and C.P. Thompson. Slowly accreting ice due to supercooled water impacting on a cold surface. Physics of Fluids, 14(1):240-256, 2002. doi: 10.1063/1.1416186. 